\title{
Reflets
}

Revue ontaroise d'intervention sociale et communautaire

\section{Résumés de mémoires de maîtrise}

Sylvie Anderson, Mélanie Audet, Jocelyne L. Bérard, Sophie Bergeron, Sylvie

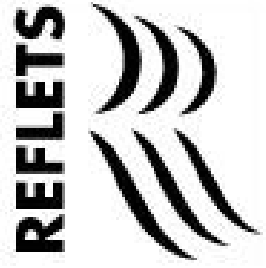
Choquette, Kimberly Fortin, Julie Gagnon, Johanne Isabelle, Marie-Josée Jobin, Rita Kendergi, Charlynne MacCharles, Aline Martel, Marie Pichette, Sophie Potvin, Julie Régimbald et Sophie Vinette

Volume 6, numéro 2, automne 2000

Problèmes sociaux en Ontario français

URI : https://id.erudit.org/iderudit/026329ar

DOI : https://doi.org/10.7202/026329ar

Aller au sommaire du numéro

Éditeur(s)

Reflets : Revue ontaroise d'intervention sociale et communautaire

ISSN

1203-4576 (imprimé)

1712-8498 (numérique)

Découvrir la revue

Citer ce document

Anderson, S., Audet, M., L. Bérard, J., Bergeron, S., Choquette, S., Fortin, K.,

Gagnon, J., Isabelle, J., Jobin, M.-J., Kendergi, R., MacCharles, C., Martel, A.,

Pichette, M., Potvin, S., Régimbald, J. \& Vinette, S. (2000). Résumés de mémoires

de maîtrise. Reflets, 6(2), 242-260. https://doi.org/10.7202/026329ar

Tous droits réservés (C) Reflets : Revue ontaroise d'intervention sociale et communautaire, 2000
Ce document est protégé par la loi sur le droit d'auteur. L'utilisation des services d'Érudit (y compris la reproduction) est assujettie à sa politique d'utilisation que vous pouvez consulter en ligne. 


\section{Résumés de mémoires de maîtrise}

\section{Le suicide chez les personnes âgées $\square$ : recherche théorique et analyse d'un programme de prévention du suicide}

Sylvie Anderson, École de service social, Université d'Ottawa

Le suicide des personnes âgées est un problème complexe et préoccupant qui attire moins d'intérêt que le suicide des jeunes dans la société canadienne. Plusieurs facteurs tels l'incompréhension du processus de vieillissement et la perception sociale négative du vieillissement peuvent contribuer à ce manque d'intérêt. Ce mémoire a pour objectif d'étudier la problématique du suicide des personnes âgées à partir d'une analyse théorique d'écrits adoptant différentes perspectives sur le sujet. Les outils théoriques ainsi identifiés ont permis d'analyser un programme d'intervention et de prévention du suicide.

La première partie du mémoire se consacre à l'analyse des statistiques sur le suicide des personnes âgées qui établit que les hommes âgés de 80 à 84 ans ont le taux de suicide le plus élevé par groupe d'âge. Quoique ces statistiques permettent d'avoir une meilleure idée de l'ampleur du suicide chez les personnes âgées, elles n'expliquent pas pourquoi les personnes âgées se suicident. Pour tenter de mieux comprendre la complexité de ce problème, quatre perspectives permettant d'expliquer le suicide ont été examinées. Ainsi, la troisième partie du mémoire porte sur l'analyse des modèles explicatifs sociologique, psychologique, épidémiologique et socio-anthropologique ayant permis, chacun 
à leur façon, d'expliquer le suicide des personnes âgées.

Cette dernière analyse nous amène à conclure que le modèle socio-anthropologique, en liant les facteurs de risque associés aux suicides des personnes âgées à leur contexte social et culturel, permet d'expliquer de façon plus complète pourquoi les personnes âgées se suicident. Par conséquent, ce modèle aurait le plus de potentiel pour élaborer un programme d'intervention pertinent et approprié. Les principes théoriques du modèle socio-anthropologique ont permis d'analyser le Programme régional de prévention du suicide d'Ottawa-Carleton et de déterminer que les systèmes de sens de la communauté et les réalités sociales et culturelles des personnes âgées d'Ottawa-Carleton n'ont pas toujours été prises en compte dans l'élaboration des lignes directrices de celui-ci.

\section{L'adoption d'enfants ayant des besoins spéciaux $\square$ : qu'en pensent les parents?}

\section{Mélanie Audet, École de service social, Université d'Ottawa}

La présente recherche exploratoire-descriptive fut effectuée par l'entremise d'un sondage auprès de 25 parents adoptifs canadiens d'enfants ayant des besoins spéciaux. Nous avons tenté d'explorer comment les parents adoptifs perçoivent la qualité de leur rôle (bénéfices et difficultés) et de mieux connaître le soutien souhaité et reçu par ces parents, ainsi que leur satisfaction quant à celui-ci. L'instrument de recherche fut adapté à partir du questionnaire SNAPS ("Special Needs Adoptive Parents Support») développé par Kramer et Houston (1998). Les résultats démontrent que ces parents adoptifs sont généralement satisfaits de leur rôle, en dépit des difficultés, sur les plans scolaire, médical et de la gestion des comportements problématiques qui y sont associés. Les parents insistent sur le fait qu'ils ont des besoins importants de soutien tels des services de répit et de garde spécialisée, des groupes de 
soutien ou d'entraide et de l'appui financier. Ces besoins persistent après que l'adoption a été finalisée, et le manque de soutien formel, tant sur le plan tangible qu'affectif, constitue une source de difficultés accrues pour les parents.

Notre étude débouche sur plusieurs implications. Sur le plan pratique, nos données suggèrent qu'il faut offrir des services après la finalisation de l'adoption, parce que les besoins spéciaux des enfants changent et que les parents ne sont pas préparés pour y répondre. Cependant, l'amélioration du soutien formel pour ces parents exige un appui financier additionnel. Ces ressources doivent aussi s'accompagner d'un appui politique des gouvernements, ainsi que d'un appui moral et social de la communauté.

Sur le plan de la recherche, il existe un besoin d'études ultérieures sur ce sujet peu exploré. Nos résultats suggèrent que les problèmes de comportement des enfants peuvent diminuer la qualité perçue du rôle parental. Ces résultats devraient être davantage explorés dans des études de plus grande envergure. De plus, il serait pertinent de procéder à une étude exploratoire auprès du personnel professionnel des agences, afin d'obtenir leur perspective au sujet des besoins non comblés de ces familles, et des obstacles à la mise sur pied de services post-adoption. Une comparaison de leur perspective avec celle des parents pourrait alimenter la réflexion sur ces questions importantes.

\section{L'implantation d'un organisme communautaire $d$ 'assistance et d'accompagnement aux plaintes dans le réseau de la santé et des services sociaux de l'Outaouais}

Jocelyne L. Bérard, École de service social, Université d'Ottawa

Le respect des droits de la personne occupe une place importante dans la société.Apprendre à les faire respecter peut être un défi de 
taille. Afin de favoriser la reconnaissance et le respect des droits de la population ayant recours aux services de santé et aux services sociaux du Québec, le législateur a prévu dans la nouvelle Loi sur les services de santé et les services sociaux (L.R.Q., chapitre 42), des mesures ainsi que des recours lorsque ces droits sont lésés.

Cette recherche touche une des mesures prévues par cette loi, soit la mise sur pied dans chaque région administrative du Québec, d'un organisme communautaire mandaté pour assister et accompagner sur demande toute personne désirant déposer une plainte relative aux services qu'elle a reçus ou qu'elle aurait été en droit de recevoir. Dans la région de l'Outaouais, le mandat est confié à l'organisme "Services conseils aux usagers des services de santé et sociaux de l'Outaouais " (SCUSSSO).

Puisque la reconnaissance des droits des usagères et des usagers est récente et, par conséquent, qu'elle a très peu bénéficié de développements théoriques, nous avons opté pour une recherche exploratoire de type descriptif. Les descriptions sous forme qualitative et quantitative ont été utilisées pour analyser la mise en oeuvre de SCUSSSO et pour suivre la première année de fonctionnement de ce nouvel organisme.

L'exploration de ce nouveau champ de pratique permet de mieux cerner le mandat d'assistance et d'accompagnement aux plaintes, d'identifier certains enjeux reliés à cette réorganisation des services et de soulever des réflexions sur la place de la travailleuse et du travailleur social dans ce nouveau service communautaire.

\section{«C'est limité mais je m'organise." Jeunes mères vivant en milieu rural $\square$ : leur perception de leurs besoins et de leurs ressources}

Sophie Bergeron, École de service social, Université d'Ottawa

Se fondant sur un devis de recherche qualitative exploratoire, la 
présente étude s'intéresse à la perception qu'ont les jeunes mères résidant en milieu rural, de leurs besoins et de leurs ressources. Nous avons voulu savoir comment ces jeunes mères définissent leurs besoins, quelles ressources elles possèdent et utilisent pour les combler et quelles sont les particularités des services et des ressources qui répondraient à leurs attentes et à leurs besoins, compte tenu notamment de leur environnement géographique. Quatre entrevues semi-dirigées et une entrevue de groupe auprès de la population cible ont permis de répondre à nos questions.

L'analyse du discours de jeunes mères a permis de dégager cinq thèmes principaux. Tout d'abord, leur choix de résider en milieu rural est marqué par le rapport étroit qu'elles entretiennent avec leur environnement social et leur milieu de vie, malgré certains désavantages liés à la ruralité. Ensuite, en dépit de leur dénuement économique, elles se battent et luttent activement par de multiples moyens de débrouillardise. Par ailleurs, la précarité pose des limites aux jeunes femmes qui ont le désir profond d'offrir à leur enfant tout ce dont il a besoin. Le rôle maternel prenant une place cruciale dans leur identité, elles tentent, malgré les contraintes, d'optimaliser le bien-être de leur enfant. D'autre part, c'est avec l'appui qu'elles trouvent auprès des proches et des ressources organisationnelles qu'elles assument leur rôle parental. Néanmoins, même si elles apprécient l'aide des organismes, leur relation avec les professionnelles se trouve ambiguë. Enfin, expertes de leur situation et actrices sociales, les jeunes mères revendiquent des services et des ressources à la mesure de leurs besoins, en liens avec leurs limites personnelles, les lacunes de leur milieu et celles des institutions. Les résultats de cette étude ouvrent la porte à des pistes de réflexion pour les pratiques et la recherche sociale en tenant compte des atouts, des attentes et des besoins des jeunes mères résidant en milieu rural. 


\section{L'état de stress post-traumatique $\square$ : quelques enjeux socio-politiques}

\section{Sylvie Choquette, École de service social, Université d'Ottawa}

Le diagnostic de l'état de stress post-traumatique est intégré depuis 1980 dans le manuel des diagnostics et statistiques des troubles mentaux (DSM-III) publié par l'Association des psychiatres américains (APA). Ce diagnostic représente l'ensemble des symptômes pouvant se développer à la suite d'un ou plusieurs événements traumatisants. Il est classé dans le DSM parmi les troubles anxieux et il est caractérisé par les souvenirs répétitifs et envahissants d'un événement extrêmement traumatisant. Des symptômes tels qu'une profonde anxiété, l'interruption de sommeil ou le retour en arrière (flashback) peuvent apparaître après un événement traumatisant, parfois même des années plus tard. Ceux-ci sont perçus en fonction de certaines conditions dont les caractéristiques personnelles et le réseau de soutien de l'individu.

Dans l'établissement du diagnostic de l'état du stress posttraumatique (ÉSPT) il est difficile d'identifier les motifs permettant d'attribuer un diagnostic psychiatrique à la souffrance sous-jacente au traumatisme. Cela a pour effet de conférer à l'individu dans une large mesure les responsabilités de ses symptômes, alors qu'un ensemble de facteurs extrinsèques à ce dernier et pouvant être observés d'un point de vue social, sont à peine considérés lors de l'évaluation psycho-médicale.

En effet, il s'agit d'examiner les motivations profondes et certains faits qui au cours de l'histoire ont favorisé le traitement de la souffrance sociale par la médicalisation. Cet examen fait ressortir plus en détails les enjeux de ce diagnostic s'appuyant sur l'approche médicale dont l'emblème, l'imposant DSM qui en est le garant reconnu pour son apport scientifique dans le domaine de la santé mentale, dissimule d'importants bénéfices financiers et de contrôle de pouvoir qui orientent la définition, les paramètres du normal, de l'excessif et du pathologique. 


\section{Les besoins et les attentes des parents qui participent à un groupe d'éducation parentale}

\section{Kimberly Fortin, École de service social, Université d'Ottawa}

Ce mémoire rend compte d'une étude portant sur les besoins et les attentes des parents qui participent à un groupe d'éducation parentale. L'objectif de cette recherche était d'abord de connaitre la façon dont les parents définissent leurs besoins et leurs attentes vis-à-vis d'un groupe d'éducation parentale. Un deuxième objectif était de vérifier si les définitions des parents correspondent à celles des intervenants et des intervenantes qui animent ces groupes. Bien qu'il existe plusieurs recherches portant sur les groupes d'éducation parentale, celles-ci offrent très peu d'éclairage sur les besoins et les attentes des parents qui participent.

La cueillette des données s'est réalisée par le biais d'entretiens semi-dirigés auprès de douze participants dont neuf parents et quatre intervenantes. Une deuxième méthode de collecte de données, soit l'observation directe, fut employée lors de trois sessions de groupe.

Les données recueillies semblent suggérer que la façon dont les parents définissent leurs besoins dépend des caractéristiques présents chez ces derniers (statut socio-économique, présence ou absence dans le marché du travail, monoparentalité, etc.). Par ailleurs, les résultats semblent également suggérer que les parents entretiennent des attentes à l'égard des groupes d'éducation parentale et ce, en dépit des résistances qu'ils pourraient éprouver. Enfin, les définitions que proposent les parents de leurs propres besoins et attentes correspondent généralement à celles proposées par les intervenantes.

Nous concluons que les groupes d'éducation parentale devraient s'adapter aux besoins des parents. Dans cette perspective, il serait utile pour les animatrices et les animateurs d'effectuer une analyse de besoin avant que débutent les sessions de groupe. Une telle initiative ne pourrait qu'augmenter l'efficacité de l'intervention. 


\section{Le traitement communautaire obligatoire $\square$ : une pratique justifiable?}

Julie Gagnon, École de service social, Université d'Ottawa

Au cours de l'hiver 2000, le gouvernement ontarien annonçait la venue prochaine des ordonnances de traitement communautaire obligatoire pour les psychiatrisés. Méthode d'intervention controversée, le traitement coercitif soulève plusieurs enjeux pratiques et éthiques, dont plusieurs liés aux droits et libertés des psychiatrisés.

Ce mémoire porte sur l'étude de la question du traitement communautaire obligatoire. Nous décrivons ce concept et le contexte au sein duquel il s'est développé. Nous identifions les partisans et les opposants au traitement coercitif, tout comme leurs arguments à l'appui, pour ensuite soulever les thèmes et les enjeux qui ressortent de ce débat. Finalement, cette étude ouvre la voie à des interventions alternatives au traitement communautaire obligatoire.

\section{L'état de stress post-traumatique et son impact sur la famille militaire}

Johanne Isabelle, École de service social, Université d'Ottawa

L'étude du trouble de l'état de stress post-traumatique (ÉSPT) fait l'objet d'un intérêt marqué depuis les dernières décennies. Néanmoins, la majorité des recherches ont été réalisées auprès des personnes souffrant de l'ÉSPT et ne se sont pas vraiment penchées avec rigueur sur la réalité des membres de la famille vivant avec ces dernières. En fait, on dispose de peu d'information sur la nature des exigences et des difficultés auxquelles ces familles 
sont confrontées. Nous jugeons important de se pencher sur cette problématique et sur son incidence sur les familles militaires, particulièrement dans un contexte où les opérations plus complexes et fréquentes de maintien de la paix, menées de front avec les compressions budgétaires et la diminution du personnel, ont eu des conséquences importantes sur les militaires canadiens et leur famille.

Ce travail se veut donc un survol de certains écrits scientifiques touchant les problèmes rencontrés par les membres des familles vivant avec un militaire affecté par l'ÉSPT. En premier lieu, il nous fait découvrir des notions théoriques entourant l'ÉSPT pour ensuite nous amener vers une réflexion sur le concept de traumatisme secondaire. Nous terminons sur des pistes d'intervention pouvant améliorer la qualité de vie des familles.

À travers les écrits, nous réalisons que les chercheures et chercheurs perçoivent davantage la famille comme une ressource naturelle. On considère que sa participation dans l'intervention du membre affecté permet de mieux comprendre ce que la personne vit afin d'être plus en mesure de l'aider dans son rétablissement. Cependant, ce que nous voulons mettre en lumière, c'est le fait qu'il est vrai de dire que la personne souffrant de l'ÉSPT a besoin de soutien, de compréhension et de patience de la part de son entourage, car il faut du temps pour guérir sa souffrance intérieure. Par contre, comme intervenant, il ne faut surtout pas oublier que les membres de la famille sont souvent eux-même affectés par la situation, au point parfois d'avoir aussi besoin d'aide. 


\section{Prostitution $\square$ : de la théorie de l'étiquetage à la pratique du vécu. La perception de cinq femmes qui font de la prostitution}

Marie-Josée Jobin, École de service social, Université d'Ottawa

Ce mémoire porte sur la perception qu'ont les femmes qui font de la prostitution de leur identité et de leur environnement de travail. Dans cette étude exploratoire de type qualitatif, nous avons tenté de mieux comprendre le processus d'étiquetage de ces femmes. Notre collecte des données a eu lieu à l'hiver 2000, avec la collaboration de cinq femmes francophones de la région de Hull-Ottawa. Chacune a participé à un entretien semi-dirigé, d'une durée de soixante minutes.

La théorie de l'étiquetage précise que c'est par un apprentissage social que les femmes ont appris leur rôle et que celui-ci les stigmatise. À partir de cette théorie, nous portons une attention particulière au rôle actif de la société dans la déviance de ces femmes et en tant que facteurs importants dans leur identification comme déviantes. Nous voyons d'une part que ce sont les groupes qui détiennent le pouvoir dans une société qui marquent les coupables et leur attribuent des sanctions, déterminées en fonction des intérêts et idéologies de ces groupes. Les femmes, quant à elles, se définissent de façon globale, autant par leurs activités reliées à la prostitution, par leurs liens individuels et sociaux que par leur socialisation.

Cette étude se centre sur le discours des femmes qui font de la prostitution. Leur histoire ressemble à des milliers de leurs consoeurs de l'Antiquité à nos jours. Du discours de ces femmes se dessinent différentes trajectoires avec une trame de fond: la peur, la honte, le courage et la détermination. Cinq thèmes s'en dégagent : le fait d'être étiquetée comme prostituée, les implications des pouvoirs, les différents acteurs des activités de la prostitution, les intervenants sociaux et la relativité du choix. 


\section{Femmes âgées immigrantes parrainées par leurs enfants $\square$ : au-delà de la dépendance}

\section{Rita Kendergi, École de service social, Université d'Ottawa}

Le parrainage des parents âgés vise à réunir les familles, objectif généralement apprécié par les personnes concernées. Pourtant, le peu de recherches qui existent sur le sujet présentent souvent les personnes âgées parrainées sous l'angle de la dépendance, de la pauvreté, de la discrimination et de l'isolement. De plus, plusieurs de ces recherches se limitent aux perceptions des chercheurs, des intervenants ou des organisations. Cette étude vise à examiner l'impact du parrainage sur des femmes âgées, en explorant le sens qu'elles donnent à leur réalité, à leurs difficultés et à leurs ressources, ainsi que les moyens auxquels elles ont recours pour modifier leur situation. Une méthode de recherche exploratoire de type qualitatif, reposant sur des entrevues à questions ouvertes auprès de quatre femmes âgées haitiennes parrainées par leurs filles, a été privilégiée afin de cerner le sens attribué par ces femmes à leur réalité sociale. L'analyse de leurs témoignages a permis de dégager le sens qu'elles donnent à leurs conditions de vie, les obstacles auxquels elles sont confrontées ainsi que les ressources et les moyens utilisés pour surmonter les défis rencontrés. L'analyse les fait apparaittre comme des « actrices » faisant appel à leur réseau informel et comptant sur leurs propres forces pour s'aider. Notre étude a permis de constater que malgré les contraintes accompagnant le statut de parrainage, les femmes disposent d'une source de revenu très modeste, grâce à l'État. Celle-ci leur permet non seulement de jouir d'une certaine autonomie financière vis-àvis de leur enfant "parrain ", mais surtout de maintenir une capacité d'échange dans leur relation avec lui. Ces constatations nous amènent à une réflexion sur les implications pour l'intervention et la recherche auprès des femmes âgées immigrantes parrainées. 


\section{L'importance de la décentration lors des interventions auprès des endeuillés, particulièrement ceux de différentes cultures}

\section{Charlynne MacCharles, École de service social, Université d'Ottawa}

Ce mémoire théorique vise à démontrer l'importance de la décentration lors de nos interventions auprès des personnes endeuillées, particulièrement celles de différentes cultures. Grâce à ce concept, nous explorons la rigidité des modèles psychologiques du deuil et nous critiquons les diagnostics liés au deuil pathologique. Certaines pratiques, de la culture chinoise en particulier, servent à illustrer la relativité culturelle du deuil. Également, certaines notions du courant anthropologique démontrent les limites culturelles des modèles psychologiques ayant une vision occidentale de la mort et du deuil. Nous nous inspirons de ce courant afin de commenter l'intolérance de la société occidentale envers la souffrance et ses répercussions.

Suite aux nombreux exemples illustrant la relativité culturelle du deuil, il devient évident que la décentration est un concept central à l'intervention auprès des endeuillés, particulièrement auprès des non-occidentaux. Quelques techniques sont proposées pour faciliter le processus de décentration : l'utilisation d'un questionnaire axé sur les perceptions, les attentes et les croyances de l'individu, une prise de conscience de sa culture et son influence, l'utilisation d'approches sensibles aux différences culturelles, la compréhension et la valorisation d'une perspective différente. Ces techniques ne représentent cependant pas une recette magique. Les intervenantes qui les adoptent avec une certaine souplesse démontreront aux usagers qu'elles ont une ouverture d'esprit à l'égard des différentes cultures et qu'elles désirent respecter leurs réactions, leurs croyances et leurs pratiques culturelles. 


\section{Féminisme et médicalisation $\square:$ l'effacement de la spécificité du corps des femmes}

\section{Aline Martel, École de service social, Université d'Ottawa}

Encore aujourd'hui, trente ans après « le début du débat entourant le problème de la désappropriation des femmes par rapport à leur corps et leur santé », la question de la santé "au féminin » est toujours au coeur des préoccupations féministes (De Koninck, Saillant et Dunnigan 1983). Entre autres, le développement des nouvelles technologies de reproduction ouvre des questions essentielles et urgentes pour le féminisme contemporain.

Ce mémoire cherche à comprendre comment la critique féministe contemporaine de la médicalisation permet de saisir le processus historique d'extension du contrôle biomédical de la reproduction et la participation des femmes à celui-ci. Notre étude met en évidence les dynamiques socioculturelles et idéologiques sous-jacentes à ces phénomènes : la conception féministe dominante du corps coïncide paradoxalement avec l'idéologie implicite au développement des technologies de la reproduction. Cette alliance contribue, d'une part, à générer une conception réductrice de la femme et de son identité et, d'autre part, à marginaliser les expériences liées à la reproduction et à la maternité.

De ce point de vue, le rapport qu'entretiennent les femmes aux sciences est problématique : il exerce un impact important sur le plan de l'identité sociale et politique de ces dernières et interroge les démocraties occidentales contemporaines. Les transformations technologiques dans le champ de la reproduction se font de manière à vouloir contrôler les femmes, à promouvoir les inégalités sociales et politiques entre les sexes ainsi que le non-respect des différences.

Cette réalité nous invite à repenser notre société et à changer les mentalités. En introduisant le concept de "différence ", l'alternative postmoderne nous permet de réfléchir à cette composante qui a été largement occultée : l'identité des femmes et son ancrage corporel. 


\section{La victimisation chez les enfants abusés sexuellement $\square$ : l'évolution de ce concept depuis les vingt dernières années}

\section{Marie Pichette, École de service social, Université d'Ottawa}

Le terme victimisation semble être utilisé de plus en plus fréquemment dans les écrits des disciplines sociales. Plusieurs définitions lui sont accordées lorsqu'il est appliqué à la problématique de l'abus sexuel pendant l'enfance. En effet, le sens que l'on donne à ce mot est intimement lié aux valeurs épistémologiques des différentes théories présentes dans le domaine du service social. Dans le but d'identifier ces théories, une grille de lecture a été appliquée aux 32 articles traitant de la victimisation des enfants abusés sexuellement tirés de la recension des écrits. Selon une classification présentée par le Conseil du statut de la femme (1995), nous avons pu relever quatre approches coexistant dans les écrits traitant de la victimisation des enfants abusés sexuellement. L'approche systémique, davantage présente, soutient que l'enfant est responsable, du moins en partie, de sa victimisation. Les tenants de la deuxième approche la plus représentée à l'intérieur de cette recension, l'approche psychologique, maintiennent que les enfants abusés sexuellement possèdent des caractéristiques spécifiques expliquant leur victimisation. La troisième approche soulevée, l'approche psychiatrique et psychanalytique, accorde une importance particulière aux conséquences de la victimisation, démontrant ainsi une utilisation plus contemporaine du terme victimisation. Pour ce qui est de la dernière approche, féministe, elle apporte une vision plus sociale de la problématique de l'abus sexuel à l'enfance par l'inclusion de variables telles les relations de pouvoir hommes-femmes et la socialisation stéréotypée du sexe féminin. À ce titre, les féministes possèdent une interprétation unique du concept de victimisation. Il est primordial pour les intervenants et intervenantes oeuvrant auprès des personnes victimes d'abus sexuel à l'enfance de 
prendre conscience des messages, implicites et explicites, véhiculés au sein de la théorie qu'ils adoptent. La responsabilisation face à l'abus sexuel peut entraver le processus de guérison chez la victime.

\section{Systèmes de gestion du risque et construction sociale de la négligence $\square$ : paradoxes et enjeux pour les services de protection de la jeunesse}

Sophie Potvin, École de service social, Université d'Ottawa

La présente étude vise à explorer, selon une perspective de construction sociale, les liens entre les systèmes de gestion du risque (SGR) appliqués au contexte québécois de protection de la jeunesse et la négligence. Notre hypothèse soutient que les SGR reproduisent et participent à la construction sociale de la négligence tout en occultant les besoins réels des enfants et de leur famille. C'est d'abord par l'analyse de la définition des SGR, $\mathrm{du}$ contexte historique et socio-politique de leur implantation et de leur application en protection de la jeunesse que nous situons, en premier lieu, la tendance des SGR à répondre davantage aux besoins organisationnels qu'aux besoins des enfants et de leur famille. Ensuite, le contexte historique de l'émergence de la négligence et son interprétation selon les différents courants d'analyse (psychologique, éco-systémique et légal) permettent de constater une prévalence de la responsabilité parentale dans la conceptualisation et l'évaluation de cette problématique.

En tant que problématique idéologiquement fondée par les structures sociales et organisationnelles, la perception de la négligence occulte les réalités sociales vécues par les familles en les tenant responsables du contexte dans lequel se construit la négligence. Par ailleurs, l'étude des SGR selon une optique de gestion de cas et de la manière dont ils dressent, dans leur contenu et leur application, une perspective idéologique de la négligence, 
permet de comprendre comment les SGR participent étroitement à la reproduction de la construction sociale de la négligence tout en répondant à des fins organisationnelles. Cette analyse permet d'argumenter que les besoins des enfants et de leur famille sont obnubilés et les interventions déroutées par la vision étroite découlant d'une approche axée sur les fondements idéologiques de la négligence et qui répond à des visées politiques et organisationnelles.

Partant du fait que les SGR sont en voie d'être implantés dans les services de protection de la jeunesse au Québec, les implications pour la pratique sont axées vers la mise en oeuvre d'une approche d'intervention qui favorise l'autodétermination des familles dans l'identification des besoins et la résolution des problèmes.

\section{L'intervention de groupe auprès des personnes atteintes d'une maladie chronique et des membres de leur famille}

\section{Julie Régimbald, École de service social, Université d'Ottawa}

Ce mémoire théorique porte sur l'intervention de groupe auprès des personnes atteintes d'une maladie chronique et de leur famille immédiate. Il s'agit d'un sujet peu exploré, surtout dans des ouvrages en français. La documentation recueillie provient des banques de données de la bibliothèque de l'Université d'Ottawa et de certaines ressources communautaires. Nous avons complété ces données théoriques avec des exemples provenant de la pratique auprès de groupes composés de personnes ayant une maladie ou un handicap et des membres de leur famille.

Les avantages principaux de l'intervention de groupe pour des personnes confrontées à une maladie chronique sont liés au fait d'aider les autres et de recevoir l'aide des pairs, à la réduction de l'isolement et à la normalisation de la situation. Les types de 
groupes les plus utilisés dans le domaine de la santé sont ceux axés sur le soutien ou sur l'apprentissage, lesquels sont dirigés par des personnes professionnelles, de même que les groupes d'entraide, qui sont dirigés par les membres. Les deux types de groupes animés par une personne professionnelle ont en commun le fait qu'ils sont généralement de type fermé (adhésion stable) et d'une durée déterminée, ce qui permet aux membres de s'engager par rapport à un but précis. Les groupes axés sur le soutien visent l'aide mutuelle, alors que ceux axés sur l'apprentissage visent plutôt l'acquisition de connaissances et de compétences. Quant aux groupes d'entraide, ils offrent des avantages semblables aux groupes axés sur le soutien, mais ils comptent davantage sur l'expertise des personnes qui ont vécu avec la difficulté.

Tous ces types de groupes ont un apport spécifique très important dans le domaine de la santé, mail il faut bien examiner les besoins de la clientèle afin de choisir celui qui est le plus pertinent. Les groupes axés sur le soutien permettent la discussion de sujets tabous, tels que les réactions émotives liées à la perte et au deuil, qui peuvent être plus faciles à aborder dans un groupe de pairs qu'avec la famille. Les groupes axés sur l'apprentissage permettent plutôt d'acquérir des compétences pour faire face aux difficultés encourues et d'augmenter les connaissances par rapport à la maladie et aux traitements, ce qui peut aider les personnes atteintes à prendre une décision éclairée par rapport à ceux-ci. Les groupes d'entraide mettent l'accent sur les forces et les capacités de gens confrontés à une maladie, ce qui les incite à utiliser l'expertise provenant de l'expérience pour aider les autres tout en s'aidant soi-même. Comme ces derniers groupes ont un apport important, il serait utile que les personnes professionnelles développent des relations de partenariat avec ceux-ci.

Il serait également pertinent d'explorer la combinaison des modèles, afin de répondre à plusieurs besoins rencontrés chez les personnes confrontées à une maladie chronique. À titre d'exemple, on pourrait réserver un certain nombre de rencontres à chacun des modèles en incluant une période de transition si nécessaire, ou encore réserver du temps pour l'éducation et le soutien à chaque réunion. Quoi qu'il en soit, il faut adapter la structure du 
groupe et considérer l'étape à laquelle les personnes se trouvent par rapport à la maladie. D'autres études sont nécessaires afin de comprendre l'apport de l'intervention de groupe dans le domaine de la santé, de même que les habiletés nécessaires à la réussite des groupes.

\section{Disciplinarisation des corps féminins et poursuite de l'idéal de la minceur $\square$ : des pratiques lourdes de sens}

Sophie Vinette, École de service social, Université d'Ottawa

Ce travail d'exploration a pour objectif d'examiner, à la lumière des études et des ouvrages qui ont été réalisées, le lien entre l'insatisfaction de l'image corporelle chez les femmes et la valorisation de la minceur dans la société nord-américaine. Pour ce faire, il s'agit d'abord d'exposer la problématique en évoquant l'ampleur du sujet, sa pertinence, de même que les concepts clefs qui y sont rattachés, soit les notions du corps, de l'image corporelle et de l'anorexie. De là émerge une recension des écrits qui donne lieu à la discussion des limites identifiées dans les études empiriques réalisées jusqu'à présent et à la présentation d'un cadre d'analyse s'appuyant à la fois sur les perspectives anthropologique, socioculturelle et féministe. Les réflexions qui découlent de cette analyse portent essentiellement sur les rôles que jouent-et les bénéfices qu'en retirent-différents systèmes ou structures sociales, par la diffusion d'un critère de beauté qui est en fait un construit social, ainsi que sur les répercussions de telles attentes sur la santé des femmes.

En s'inspirant d'une approche foucaldienne, le présent travail cherche également à enrichir l'analyse du phénomène par l'exploration plus poussée de la notion de pouvoir, de la disciplinarisation des corps féminins et du souci de soi. Ce faisant, le travail s'attarde à l'étendue et à la variété des pratiques disciplinaires de même qu'aux phénomènes de normalisation et 
d'intériorisation qui les nourrissent. Enfin, les implications pour l'intervention sont mises en évidence et diverses pistes sont offertes à la lumière des conclusions tirées. 